tial for replacing EtO; however, sterilization manufacturers must refine their processes to enhance their microbiocidal efficacy. Equally important, device manufacturers must design their instruments to allow proper cleaning and effective sterilization.

\section{REFERENCES}

1. Alfa MJ, DeGagne P, Olson N, Puchalski T. Comparison of ion plasma, vaporized hydrogen peroxide and $100 \%$ ethylene oxide sterilizers to the $12 / 88$ ethylene oxide gas sterilizer. Infect Control Hosp Epidemiol 1995;17:92-100.
2. Environmental Protection Agency. Protection of Stratospheric Ozone; Proposed Rule. Federal Register. May 12, 1993.

3. Schneider PM. Low-temperature sterilization alternatives in the 1990s. Tappi Journal 1994;77:115-119.

4. Gross D. Ethylene oxide sterilization and alternative methods. Surgical Services Management 1995;1:16-17.

5. Food and Drug Administration, Division of General and Restorative Devices. Guidance on Premarket Notification $[510(\mathrm{k})]$ Submissions for Sterilizers Intended for Use in Health Care Facilities. Washington, DC: FDA; March 1993.

6. Holler C, Martiny H, Barbel C, Rüden H, Gundermann K. The efficacy of low-temperature plasma (LTP) sterilization, a new sterilization technique. Zbl Hyg 1993;194:380-391.

\title{
Smoke Tubes Not Reliable for Negative-Pressure Monitoring
}

\section{by Gina Pugliese, RN, MS Medical News Editor}

It is recommended that TB isolation rooms be monitored regularly for the direction of airflow to assure that there is negative pressure in relation to the corridor. Researchers at the Montreal Chest Research Institute in Montreal, Quebec, Canada, developed a simple method to measure airchange rates and direction of airflow in patient-care areas. Pure carbon dioxide $\left(\mathrm{CO}_{2}\right)$ was released at 13.5 $\mathrm{L} / \min$ for 5 minutes, then measured for 30 minutes within the room and outside in the hallway with a $\mathrm{CO}_{2}$ reading instrument. Smoke tubes also were used to measure direction of airflow. If doors and windows were opened, there were significant changes in air-change rates and airflow direction. Smoke-tube measurements were inconsistent, agreed poorly with evidence of $\mathrm{CO}_{2}$ movement from room to hall, and were affected strongly by room-to-hallway temperature differentials.

The authors concluded that smoke tubes, although inexpensive and simple, are unreliable and that $\mathrm{CO}_{2}$ release and measurement pro- vides more accurate measurement of air-change rates and airflow direction and the effect of door or window manipulation. The authors note that $\mathrm{CO}_{2}$ is safe, nontoxic, and inexpensive, and the technique for measuring $\mathrm{CO}_{2}$ is fairly simple. A direct-reading $\mathrm{CO}_{2}$ detector is needed and costs approximately $\$ 1,500$.

From: Menzies R, Schwartzman $\mathrm{K}$, Loo V, Pasztor J. Measuring ventilation of patient care areas in hospitals: description of a new protocol. Am J Respir Crit Care Med 1995; (152):1992-1995. 